\title{
Modeling Crystallization Dynamics when the Avrami Model Fails
}

\author{
TERRY GOUGH ${ }^{\mathrm{a}}$ and REINHARD ILLNER ${ }^{\mathrm{b}, *}$ \\ ${ }^{a}$ Department of Chemistry, University of Victoria, P.O. Box 3065, Victoria, B.C. V8W 3V6, Canada, \\ e-mail: gought@uvvm.uvic.ca; ${ }^{b}$ Department of Mathematics and Statistics, University of Victoria, \\ P.O. Box 3045, Victoria, B.C. V8W 3P4, Canada
}

(Received 13 August 1997; In final form 1 December 1998)

\begin{abstract}
Recent experiments on the formation of crystalline $\mathrm{CO}_{2}$ from a newly discovered binary phase consisting of $\mathrm{CO}_{2}$ and $\mathrm{C}_{2} \mathrm{H}_{2}$ at $90^{\circ} \mathrm{K}$ fail to be adequately simulated by Avrami equations. The purpose of this note is to develop an alternative to the Avrami model which can make accurate predictions for these experiments. The new model uses empirical approximations to the distribution densities of the volumes of three-dimensional Voronoi cells defined by Poisson-generated crystallization kernels (nuclei). Inside each Voronoi cell, the growth of the crystal is assumed to be linear in diameter (i.e., cubic in volume) until the cell is filled by the $\mathrm{CO}_{2}$ crystals and the $\mathrm{C}_{2} \mathrm{H}_{2}$ (thought of as a waste product). The cumulative growth curve is computed by averaging these individual growth curves with respect to the distribution density of the volumes of the Voronoi cells. Agreement with the experiments is excellent.
\end{abstract}

Keywords: Crystallization, Avrami model, binary phase

\section{INTRODUCTION}

Crystal growth is of obvious significance in semiconductor design. The classical approach to model the formation of crystals is based on a probabilistic argument and known as the Avrami model [4]. We review the Avrami model in Section 2 but state here that the Avrami equations predicting conversion to crystal as a fraction of total available volume are

$$
\varphi(t)=1-\mathrm{e}^{-K t^{n}}
$$

where $K$ and $n$ are parameters.

The purpose of this note is to offer an alternative to the Avrami model based on statistical properties of Voronoi diagrams. It is straightforward to see (and well-known) that the Voronoi diagrams

*Corresponding author. e-mail: rillner@math.uvic.caz 
associated with the crystallization kernels (impurities referred to as nuclei) are important for the crystallization process: Crystals will form as spherical globules and grow linearly with time in diameter, hence cubic in volume, around the impurities, until these globules impinge into each other; the latter occurs exactly at the interfaces defining the Voronoi diagram, because these interfaces are defined as being equidistant to the two closest nuclei. See Figure 1.

One characteristic of Avrami equations is that they predict that regardless of what $n$ is, the length of time required to convert the first half of the reactant to product is shorter than the time required for the second half of the conversion. One can argue that the slowing down occurs when

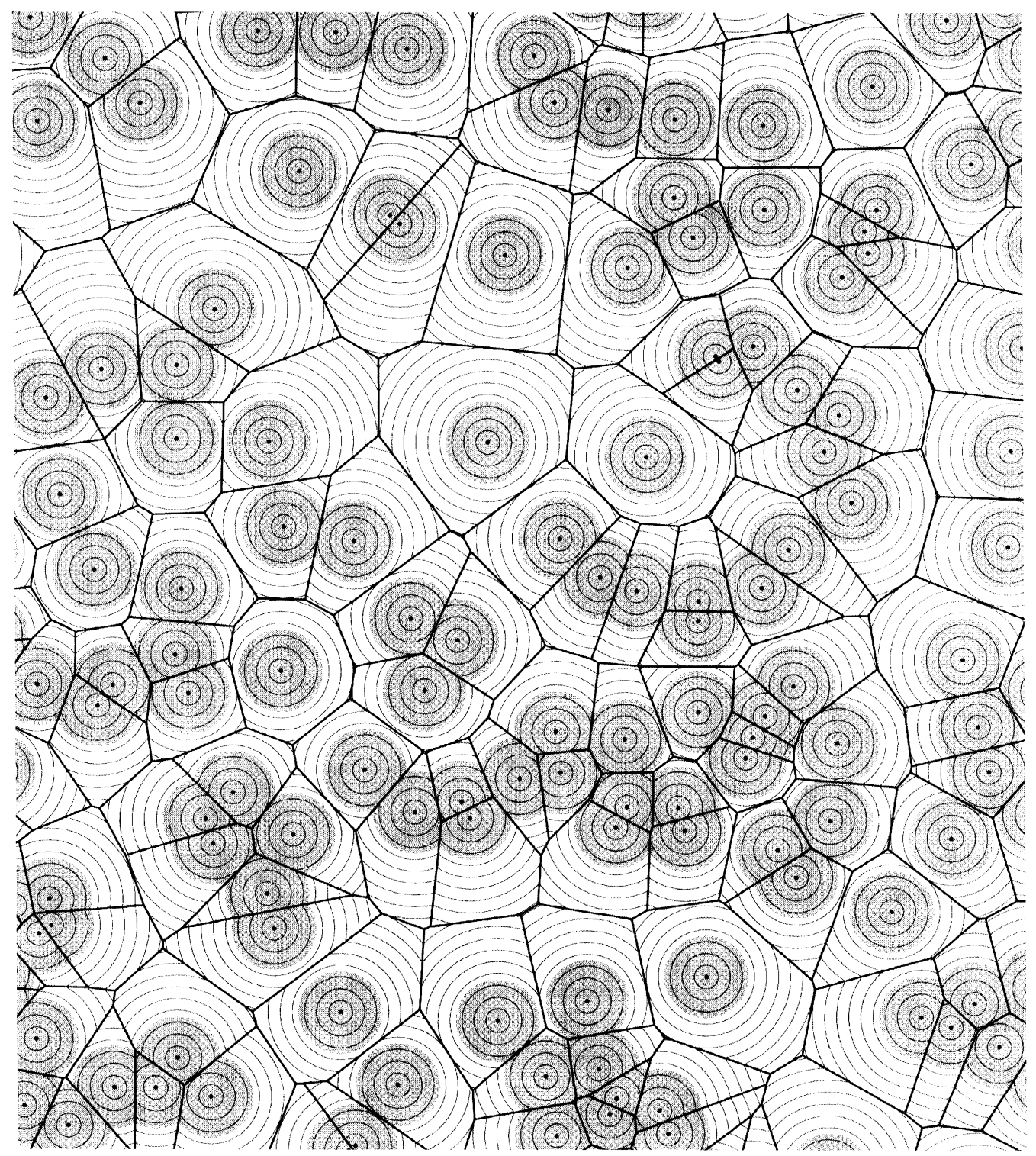

FIGURE 1 Voronoi cells. 
expanding reaction fronts collide at the boundary separating adjacent Voronoi cells. After such collisions, the surface at which conversion occurs no longer grows as the cube of time.

The experiment which inspired us to look for an alternative to the Avrami model was the formation and disintegration of a metastable crystalline binary phase $\mathrm{CO}_{2} \cdot \mathrm{C}_{2} \mathrm{H}_{2}$ under suitable conditions: Mixtures of these two gases are expanded through a nozzle onto a zinc selenide window which is mounted in the beam of an FTIR spectrometer and maintained at $90 \mathrm{~K}$. The pulse deposits a film approximately 200 molecules thick. The obtained spectra depend markedly on the conditions of the expansion. In general, absorptions characteristic of crystalline $\mathrm{CO}_{2}$ and crystalline $\mathrm{C}_{2} \mathrm{H}_{2}$ are observed together with new features assigned to a mixed phase. For 1:1 mole fractions the absorptions assigned to the pure phases are absent, and it was found that the intensity ratio of the new features is independent of the mole fraction of $\mathrm{CO}_{2}$. These observations establish the stoichiometry of the new phase as $\mathrm{CO}_{2} \cdot \mathrm{C}_{2} \mathrm{H}_{2}$ (for more details, see [3]).

It was then observed from spectra recorded over a period of 5 hours that the $\mathrm{CO}_{2} \cdot \mathrm{C}_{2} \mathrm{H}_{2}$ decomposes into $\mathrm{CO}_{2}$ and $\mathrm{C}_{2} \mathrm{H}_{2}$. Experimental data for fractional conversion versus time, based on spectral intensities, are given in Figure 2.

The $\mathrm{CO}_{2}$ formed has a sharp spectrum indicating crystallinity, whereas the $\mathrm{C}_{2} \mathrm{H}_{2}$ has broad absorptions indicating a more amorphous state. It was established [3] that the reaction is a solid state transformation. The plot in Figure 2 is sigmoidal, suggesting that an Avrami analysis might be appropriate. However, the decomposition does not show the characteristic slowing down discussed earlier: Rewriting (1.1) as

$$
1-\varphi(t)=\mathrm{e}^{-K t^{n}}
$$

taking logarithms

$$
-\ln (1-\varphi(t))=K t^{n}
$$

and taking logarithms again yields

$$
\ln (-\ln (1-\varphi(t)))=\ln K+n \ln t .
$$

So the left-hand side of (1.2) is a linear function in $\ln t$. However, plotting $\ln (-\ln (1-$ converted fraction)) versus $\ln t$ yields the curve given in Figure 3, which is obviously not linear.

The curve in Figure 3 indicates that $n$ increases smoothly from 1 at the beginning to 4 at its conclusion. A least squares fit to the overall data provided a value of $n=3.3$.

The basic idea put forward in this paper is to produce approximations to the crystal growth curves (as a fraction of total volume) by averaging the growth curves associated with the individual Voronoi cells with respect to the statistical properties of a Voronoi diagram for which the impurities are generated by a Poisson process. In the absence

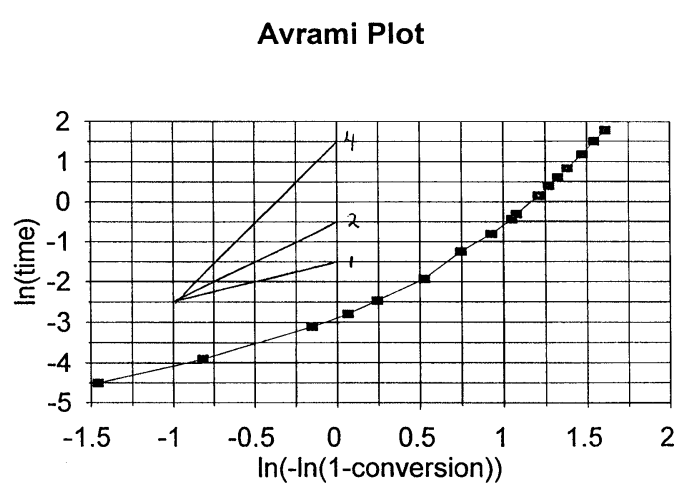

FIGURE 3 Testing the Avrami model.

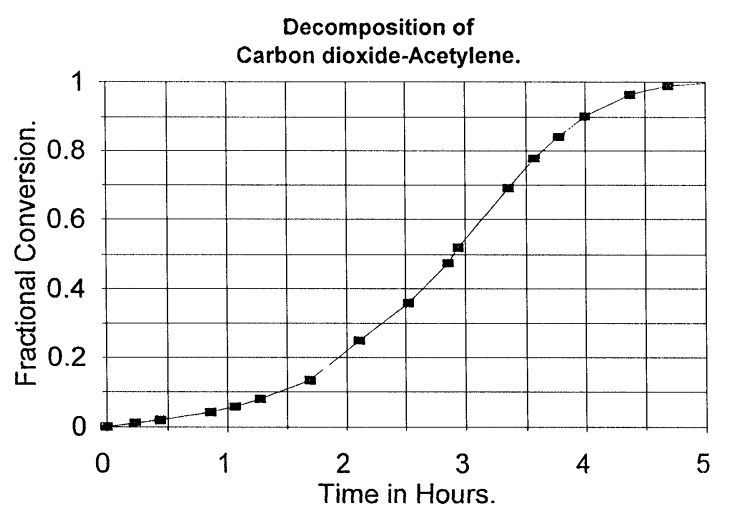

FIGURE 2 The experiment. 
of other information, assuming that the impurities are Poisson-distributed is natural.

Our approach contains two inherent difficulties:

1. The growth curve of the piece of crystal inside a Voronoi cell depends on the geometry of that cell. The volume growth is assumed to be cubic with time until a boundary is reached, then slow down because there cannot be any growth beyond that boundary, and stop completely once all the corners of the cell are filled. The end phase of the growth will therefore vary from cell to cell.

2. While the statistical properties of Voronoi cells are a widely investigated topic [1], the exact probability distribution of the volumes of Voronoi cells associated with Poisson-distributed points is not known. In two dimensions, there are a lot of empirical studies and matches to these studies with various ad hoc approximations, like Gamma distributions, Maxwell distributions or lognormal distributions. We refer to [1] for details. An empirical numerical study on the volume distribution of three-dimensional Voronoi cells generated by a Poisson process is given in [2].

We cannot deal with these difficulties rigorously; we avoid them by making simplifying assumptions. As for 1., we simply assume that growth proceeds as $k t^{3}$ until the cell is full, i.e., for a cell of volume $a>0$ the growth curve is given by

$$
g(a, t)=\left\{\begin{array}{cl}
k t^{3}, & 0 \leq t \leq(a / k)^{1 / 3} \\
a, & (a / k)^{1 / 3} \leq t
\end{array}\right.
$$

Here, $k$ denotes the (linear) growth rate of the radius of the globule forming inside the cell.

This assumption is clearly simplistic, but the error is small for Voronoi cells which are regular in the sense that they are well approximated by spheres (we refrain from trying to produce a rigorous approximation criterion). A mitigating factor in this context is the observation that the crystallization process involves two products, namely the pure $\mathrm{CO}_{2}$ crystals, which grow from the nuclei, and the more amorphous $\mathrm{C}_{2} \mathrm{H}_{2}$, which will accumulate at the boundaries and in the corners of the Voronoi cells.

As for 2., we tried a number of density distribution functions for the volume of Poisson-generated Voronoi cells. It will be shown below that there is a choice of a probability density which reproduces the Avrami model. However, using semi-empirical approximations as suggested in [1] and [2] produced much better agreement with our experimental data.

The plan of this paper is as follows. We briefly review the Avrami model in Section 2. In Section 3 , we establish a general formula for growth curves by averaging over the curves given in (1.3) with respect to $a$. Several examples are discussed, and one of them reproduces the Avrami model. For other semi-empirical approximations to the Voronoi cell volume distribution, the general formula yields integrals which we evaluated by using the MAPLE symbolic computation program. We present the best match we obtained in this way to a data set produced in [3] in Section 4.

\section{REVIEW OF THE AVRAMI MODEL}

A direct derivation of the Avrami model from probabilistic considerations works as follows.

Assume that $N$ nuclei are equidistributed distributed in a (macroscopic) Volume $V>0$. Choose an arbitrary but fixed reference point $P$ and let $X$ be the distance to the nearest nucleus. $X$ is a random variable, and

$$
P\{X>x\}=\left(1-\frac{\frac{4}{3} \pi x^{3}}{V}\right)^{N}
$$

so the cumulative distribution function of $X$ is

$$
F_{N}(x)=P\{X \leq x\}=1-\left(1-\frac{\frac{4}{3} \pi x^{3}}{V}\right)^{N} .
$$

In the limit $N \rightarrow \infty, V \rightarrow \infty$ such that $(N / V) \rightarrow$ $\lambda>0$ (intensity of the Poisson process), $F_{N}$ 
converges to

$$
F(x)=1-\mathrm{e}^{-\frac{4}{3} \pi \lambda x^{3}}
$$

If crystalline globules grow at speed $v$ from each nucleus, this calculation translates directly into the probability that crystallization at time $t$ has reached the point $P$ :

$$
\varphi(t)=P\{X<v t\}=1-\mathrm{e}^{-\frac{4}{3} \pi \lambda v^{3} t^{3}} .
$$

\section{AVERAGED GROWTH CURVES}

We now present an alternative approach which contains the Avrami equation (2.2) as a special case. Suppose that $f(a), a \geq 0$, is the density distribution function for the volume of the Voronoi cells (in 3D) associated with Poisson distributed nuclei. We average the individual growth curves given by (1.3) with respect to $f$ and compute a macroscopic, observable growth curve as

$$
\begin{aligned}
\varphi(t) & =\int_{0}^{\infty} g(a, t) f(a) d a \\
& =\int_{0}^{k t^{3}} a f(a) d a+k t^{3} \int_{k t^{3}}^{\infty} f(a) d a
\end{aligned}
$$

The upper limit $\infty$ in the integrals on the right is used because we can assume that the largest theoretically possible Voronoi cell is of macroscopic scale relative to the typical one. The two-dimensional analogue to (3.1) is

$$
\varphi(t)=\int_{0}^{k t^{2}} a f(a) d a+k t^{2} \int_{k t^{2}}^{\infty} f(a) d a
$$

Formula (3.1) is offered as an alternative to the Avrami equation (2.2). Of course, we have to know what $f$ is in order to produce a usable equation. Unfortunately, the true $f$ is not known (but an object of study, see [1]); we discuss a few examples with ad hoc choices for $f$. The last example is then matched to experimental data in the next section.
Example 3.1 To get a feeling for the type of curves which (3.1) produces, we assume that $f$ is the equidistribution on an interval $[0, A]$, with $A>0$, i.e.,

$$
f(a)=\frac{1}{A} \chi_{[0, A]}(a) .
$$

For this $f$, the integration in (3.1) can be done explicitly, and yields

$$
\varphi(t)= \begin{cases}k t^{3}-\frac{1}{2 A} k^{2} t^{6} & \text { for } k t^{3}<A \\ \frac{A}{2} & \text { for } k t^{3} \geq A\end{cases}
$$

These curves already display the correct logistictype growth, and they show the kind of asymmetry about the half-life point (i.e., the point where half of the substance has crystallized) which was seen in the experiment described in [3].

Example 3.2 The equidistribution used in Example 3.1 is clearly not a very intelligent guess for the volume distribution of Voronoi cells. Let us make a more systematic attempt, following the derivation of the Avrami model in Section 2.

Assume that $N$ nuclei are Poisson distributed in a (macroscopic) Volume $V>0$. Choose one of these nuclei arbitrary but fixed and let $X$ be the distance to its nearest neighbor. $X$ is a random variable, and

$$
P\{X>x\}=\left(1-\frac{\frac{4}{3} \pi x^{3}}{V}\right)^{N-1}
$$

so the cumulative distribution function of $X$ is

$$
F_{N}(x)=P\{X \leq x\}=1-\left(1-\frac{\frac{4}{3} \pi x^{3}}{V}\right)^{N-1}
$$

hence the density $f_{N}(x)$ is

$$
f_{N}(x)=F_{N}^{\prime}(x)=4 \pi \frac{N-1}{V} x^{2}\left(1-\frac{4}{3} \pi \frac{x^{3}}{V}\right)^{N-2}
$$

In the limit $N \rightarrow \infty, V \rightarrow \infty$ such that $(N / V) \rightarrow$ $\lambda>0$ (intensity of the Poisson process), $f_{N}$ 
converges to

$$
f(x)=4 \pi \lambda x^{2} \mathrm{e}^{-\frac{4}{3} \pi \lambda x^{3}}
$$

and $F_{N}$ to

$$
F(x)=1-\mathrm{e}^{-\frac{4}{3} \pi \lambda x^{3}}
$$

We use these to compute the density of the random variable $S:=$ volume of the sphere with radius $X / 2$; this is the largest sphere which will fit into the Voronoi cell centered at the chosen nucleus. Clearly,

$$
\begin{aligned}
P\{S \leq s\} & =P\left\{\frac{\pi}{6} X^{3} \leq s\right\} \\
& =P\left\{X \leq\left(\frac{6 s}{\pi}\right)^{\frac{1}{3}}\right\} \\
& =1-\mathrm{e}^{-8 \lambda s} .
\end{aligned}
$$

The associated density is

$$
g(s)=8 \lambda \mathrm{e}^{-\lambda s} .
$$

We note for later reference that

$$
E(S)=\frac{1}{8 \lambda}
$$

and hence the expected volume occupied by all such spheres around all nuclei is $(N / 8 \lambda)=(V / 8)$. So $7 / 8$ of the available volume belongs to corners of Voronoi cells in this sense.

If we use $g$ as obtained in (3.2) in the formula (3.1), we find

$$
\begin{aligned}
\varphi(t) & =8 \lambda \int_{0}^{k t^{3}} a \mathrm{e}^{-8 \lambda a} d a+8 \lambda k t^{3} \int_{k t^{3}}^{\infty} \mathrm{e}^{-8 \lambda a} d a \\
& =\frac{1}{8 \lambda}\left[1-\mathrm{e}^{-8 \lambda k t^{3}}\right]
\end{aligned}
$$

and modulo a normalizing factor this is the Avrami equation (2.2) rediscovered.

Example 3.3 The last example which we present uses the density $f(x)=\alpha x^{2} \mathrm{e}^{-\beta x^{2}}$, where $\alpha, \beta$ are parameters; they are linked because $f$ has to be a probability density, so $f$ depends on only one free parameter. We arrive at the formula

$$
\varphi(t)=\alpha \int_{0}^{k t^{3}} x^{3} \mathrm{e}^{-\beta x^{2}} d x+\alpha k t^{3} \int_{k t^{3}}^{\infty} x^{2} \mathrm{e}^{-\beta x^{2}} d x
$$

The first integral is easy to compute; the second requires the error function to be expressed in closed form. Symbolic evaluation of $\varphi(t)$ is easily done by using a symbolic computation package like, e.g., MAPLE.

We experimented with many other densities. The three given above were chosen for the following reasons: 3.1 is the simplest example; 3.2 reproduces the Avrami equation; and 3.2 gave us the best agreement with experiments, as we show in the final section.

\section{COMPARISON WITH THE EXPERIMENT}

The density from Example 3.3, with $\beta=1$, gave the match to the data points which we present in Figure 4.

The agreement with the experimental data is striking, suggesting that the assumptions underlying the modeling process are realistic.

Infrared spectroscopy of the reactant and product phases shows that the reactant phase and the product carbon dioxide are both highly crystalline

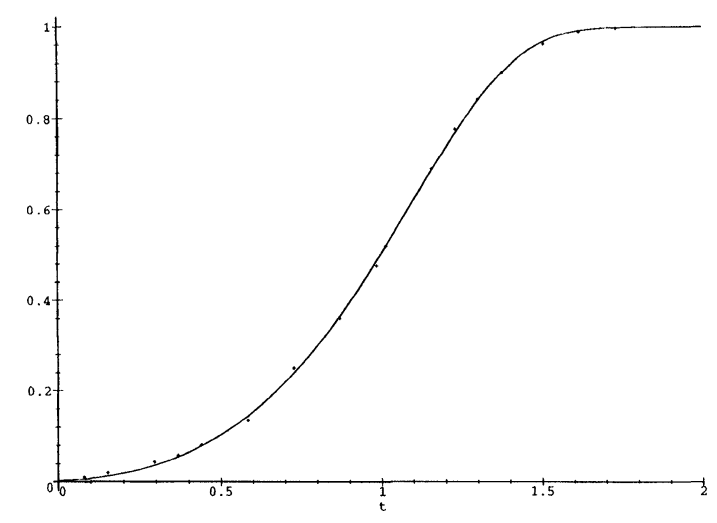

FIGURE 4 Matching the data with the alternative model. 
while the product acetylene phase is amorphous. These observations are consistent with the product consisting of crystals of carbon dioxide embedded in a less rigid acetylene matrix. This means that each Voronoi cell will only be half full of rigid material and this material may be free to move.

Also, we point out that the density $f(x)=$ $\alpha x^{2} \mathrm{e}^{-\beta x^{2}}$ appears to be a good approximation for the density distribution of the volumes of Voronoi cells associated with Poisson-distributed nuclei (see [2] for an empirical study).

Wunderlich [4] has reported experimental calorimetric data for the crystallization of a copolymer of ethylene terephthalate and ethylene sebacate. This system resembles the present one in that crystallization does not proceed to completion and so the final product consists of crystalline regions imbedded in a less rigid amorphous matrix. The data were subjected to an Avrami analysis and $n$ was found to be 3.2. Furthermore, the data deviated from the expectations of the model in the same sense as the present data. We conclude that the present model would better describe the crystallization of the copolymer, and presumably many other systems.

\section{Acknowledgement}

This research was supported by grants from the Natural Sciences and Engineering Research Council of Canada. The authors are grateful to Dennis Manke, who produced Figure 1 with his Voronoidiagram producing software.

\section{References}

[1] Okabe, A., Boots, B. and Sugihara, K. (1992). Spatial Tesselations: Concepts and Applications of Voronoi Diagrams, J. Wiley.

[2] Quine, M. P. and Watson, D. F. (1984). Radial Generation of $n$-Dimensional Poisson Processes, J. Appl. Prob., 21, $548-557$.
[3] Rowat, T. (1997). Stoichiometry and Stability of Binary Phase Crystals formed between Acetylene and Nitrous Oxide/Carbon Dioxide, Ph.D. Thesis, University of Victoria.

[4] Wunderlich, B. (1976). Macromolecular Physics, Vol. 2: Crystal Nucleation, Growth, Annealing, p. 132 ff. Academic Press.

\section{Authors' Biographies}

Terry Gough was born on October 12, 1939 in Portsmouth, England. He attended the Portsmouth Grammar School, and the University of Leicester where he obtained his B.Sc. and Ph.D. The latter degree was obtained under the supervision of Professor M.C.R. Symons, ostensibly studying ion solvation and association using ultraviolet spectroscopy. In 1965 he joined the Department of Chemistry at the University of Waterloo to establish a nuclear magnetic resonance facility. In 1976 he began a collaboration with Giacinto Scoles which lead to the formation of the Centre for Molecular Beams and Laser Chemistry at the University of Waterloo. In 1989 he moved to the University of Victoria as Chairman of Chemistry where he is currently studying vibrational overtones of molecular beams and the FTIR spectroscopy of large molecular clusters.

Reinhard Illner was born on January 2, 1950 in Wabern, Germany. He studied Mathematics at the Universities of Heidelberg, Berkeley and Bonn and obtained his Ph.D. under the supervision of Professor J. Frehse in Bonn in 1976. He has held positions at the Universities of Bonn, Kaiserslautern, Duke University and the University of Victoria, where is currently full professor and chair of the Mathematics and Statistics department. His main field of research is the mathematical theory of Nonequilibrium Statistical Mechanics. He is co-author of the monograph "The Mathematical Theory of Dilute Gases", which appeared in 1994. 

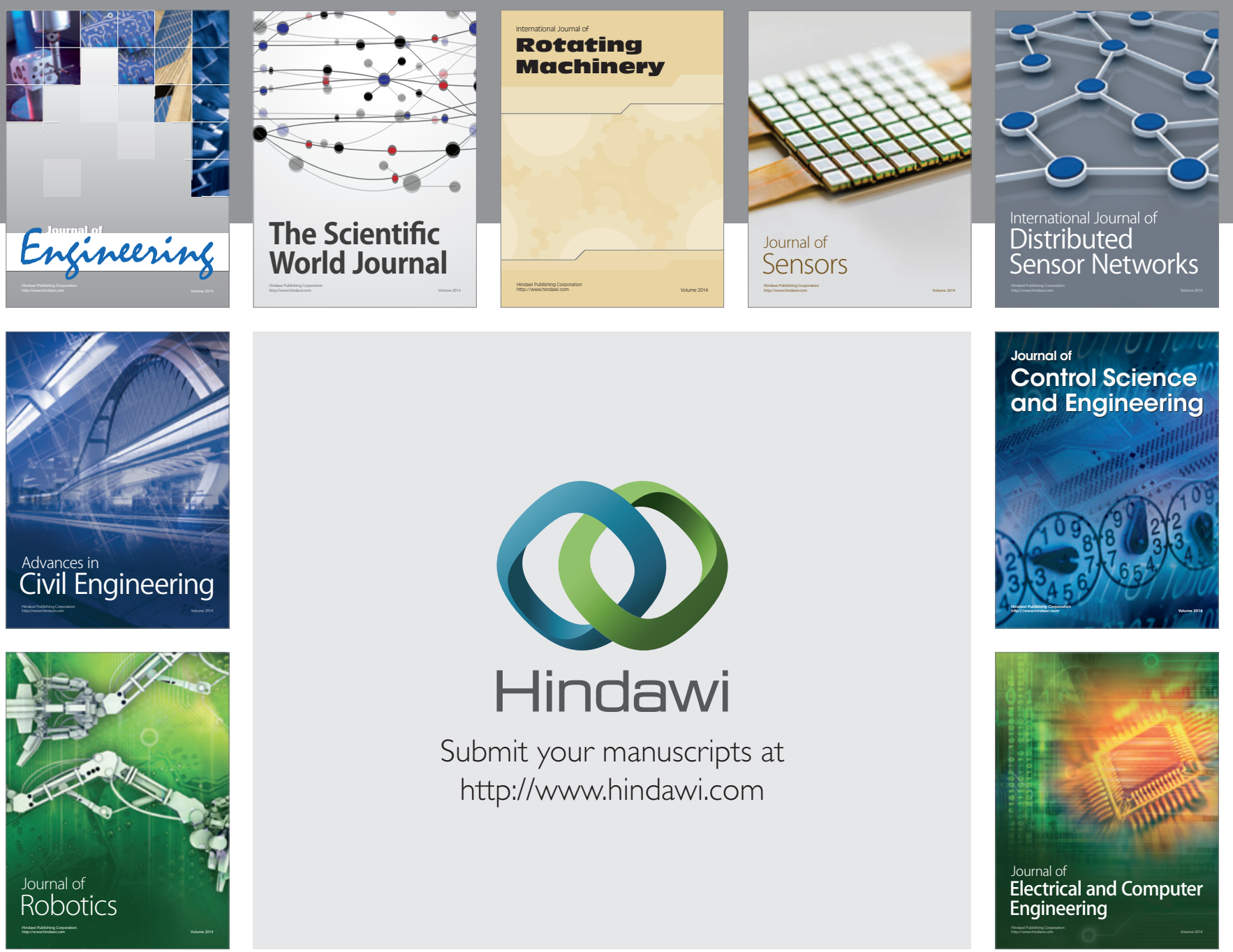

Submit your manuscripts at

http://www.hindawi.com
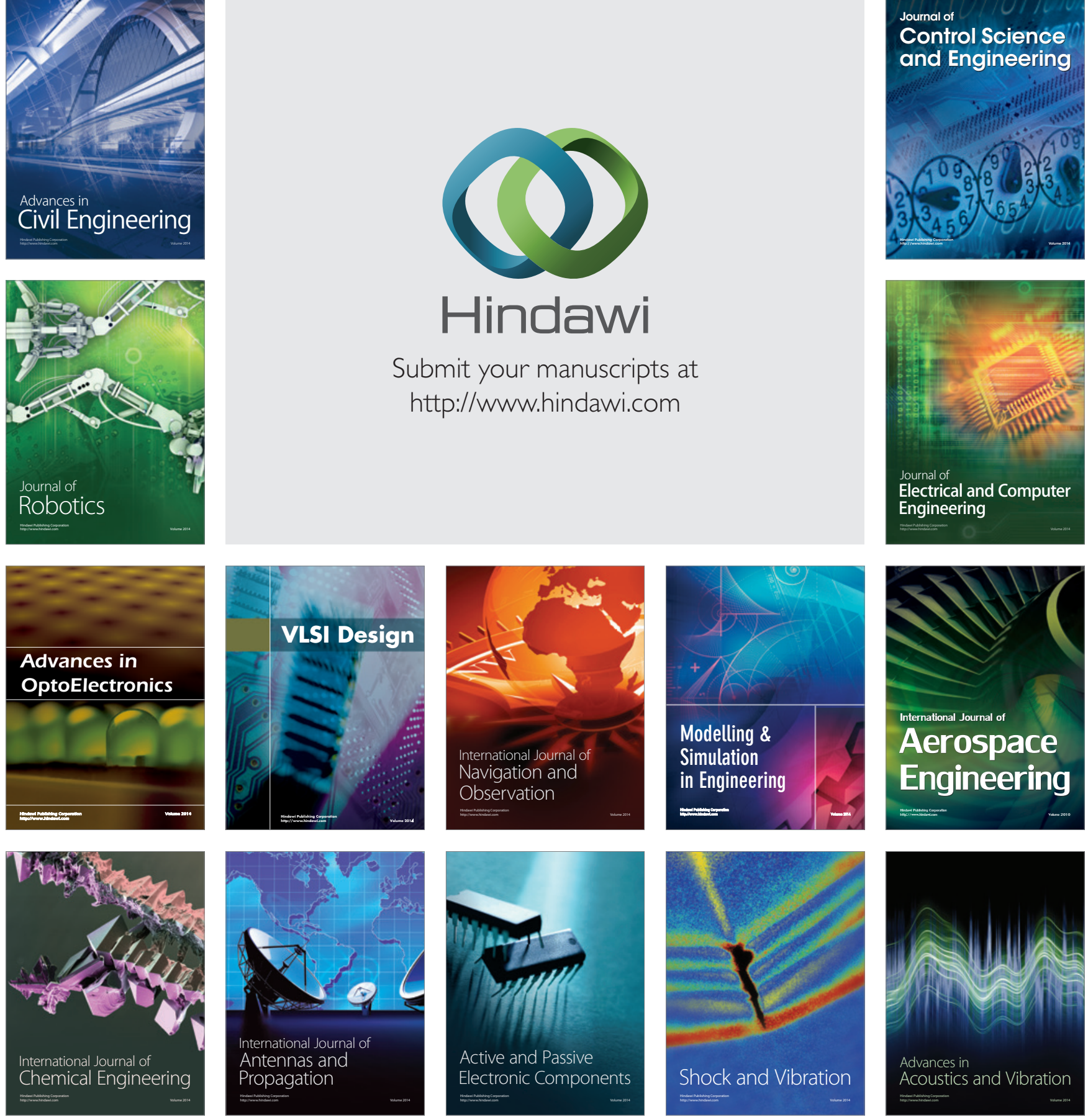